\title{
Exploring Collaborative HPC Visualization Workflows using Vislt and Python
}

\author{
Hari Krishnan ${ }^{\mathbb{I} *}$, Cyrus Harrison ${ }^{\ddagger}$, Brad Whitlock ${ }^{\ddagger}$, David Pugmire ${ }^{\S}$, Hank Childs $\|$ \\ http://www.youtube.com/watch?v=ei_pFi2xOUc
}

\begin{abstract}
As High Performance Computing (HPC) environments expand to address the larger computational needs of massive simulations and specialized data analysis and visualization routines, the complexity of these environments brings many challenges for scientists hoping to capture and publish their work in a reproducible manner.

Collaboration using HPC resources is a particularly difficult aspect of the research process to capture. This is also the case for HPC visualization, even though there has been an explosion of technologies and tools for sharing in other contexts.

Practitioners aiming for reproducibility would benefit from collaboration tools in this space that support the ability to automatically capture multi-user collaborative interactions. For this work, we modified Vislt, an open source scientific visualization platform, to provide an environment aimed at addressing these shortcomings.

This short paper focuses on two exploratory features added to Vislt:

1. We enhanced Vislt's infrastructure expose a JSON API to clients over WebSockets. The new JSON API enables Vislt clients on web-based and mobile platforms. This API also enables multi-user collaborative visualization sessions. These collaborative visualization sessions can record annotated user interactions to Python scripts that can be replayed to reproduce the session in the future, thus capturing not only the end product but the step-by-step process used to create the visualization.

2. We have also added support for new Python \& R programmable pipelines which allow users to easily execute their analysis scripts within Vislt's paralle infrastructure. The goal of this new functionality is to provide users familiar with of Python and $\mathrm{R}$ with an easier path to embed their analysis within Vislt.

Finally, to showcase how these new features enable reproducible science, we present a workflow that demonstrates a Climate Science use case.
\end{abstract}

Index Terms-python, reproducibility, collaboration, scripting

\section{Introduction}

Reproducibility is one of the main principles of the scientific method.

Without reproducibility, experimental trials that confirm or deny a given hypothesis cannot be confirmed by other scientists, potentially creating concerns about the validity of initial results.

Visualization often plays a role in the scientific method; when exploring data sets, scientists form hypotheses about phenomena

\author{
* Corresponding author: hkrishnan@lbl.gov \\ Il Lawrence Berkeley National Laboratory \\ + Lawrence Livermore National Laboratory \\ $\S$ Oak Ridge National Laboratory \\ II Lawrence Berkeley National Laboratory/The University of Oregon
}

Copyright (C) 2013 Hari Krishnan et al. This is an open-access article distributed under the terms of the Creative Commons Attribution License, which permits unrestricted use, distribution, and reproduction in any medium, provided the original author and source are credited. in the data, design experiments by setting up visualization parameters, and then carry out the experiment by applying visualization algorithms. The resulting visualizations then confirm or deny each hypothesis. However, since this process is regularly carried out in an ad hoc manner and in rapid succession, reproducibility is often a secondary concern.

Consequently, the outputs from visualization and analysis routines often lack the information about how they were generated, and thus how to interpret the results.

In favorable circumstances, the initial scientist performing the analysis either took notes or remembers the details of the experiment, and theoretically would be able to reproduce it. But following scientists regularly do not have this information. Although they can view the resulting visualizations, and make educated guesses about how the data was processed, reproducing the result is very difficult. This is particularly true because visualization routines have many 'knobs' that control how they execute.

Beginning approximately one decade ago, the visualization community increased its emphasis on including provenance as part of the visualization process.

For example, the VisTrails system [silva2007prov], an early provenance advocate, produced the necessary information to recreate everything about a given visualization.

This represented a leap forward in the problem, since the ad hoc and rapid nature of visualization-based exploration could now be automatically accounted for.

However, provenance is still far from being commonplace, and only rarely do scientists broadcast their exact steps to create their results.

Further, provenance is only one component of the larger problem. Knowing the parameters that went into a visualization is important, but these parameters are much less meaningful when the program used to generate the results is no longer available. This is especially problematic when 'one-off' programs are generated to create a specific visualization, a common scenario when people are performing novel analysis. After one-off programs generate the necessary visualizations, their code often quickly atrophies or is lost altogether. Finally, such programs are rarely accessible to following scientists who recreate the experiment.

Following these observations, the research described in this paper depends on the following premises:

1. Enduring visualization frameworks are crucial for maintaining reproducibility.

- We also note that focusing on a single application-as opposed to many one-offs for 
many problems - allows for significantly more resources to be allocated to development, allowing the application to be maintainable, reliable, sharable, and to have important reproducibility features, i.e., provenance.

2. These frameworks must provide constructs that enable novel and complex analyses.

With this research, we explored adding a flexible, Pythonbased infrastructure to an existing visualization framework. Our Python system is made up of rich, composable operations that enable the development of new, novel analyses which can then be reincorporated into the visualization framework. This approach enables the specialized analysis typically reserved for one-off applications to be handled within one application, significantly increasing the capabilities available to scientists. In this paper, we describe the system, as well as a use case in climate science.

Finally, leading-edge simulation science increasingly involves large teams with diverse backgrounds, and these teams need to be able to analyze data in collaborative settings. But collaborative analysis complicates the provenance tracking that is necessary for reproducibility. Our system is able to perform this tracking and we describe how it functions.

\section{Related Work}

There is growing interest in the practice of reproducible research for simulations. Open source software, virtualization, and cloud computing platforms have enabled workflows that can be adopted by scientific peers with very low barriers to entry [res_cloud], [web_repro]. Increased interest in reproducibility also is driven by notable research retractions such as Herndon, Ash and Pollin's re-analysis [herndon_debt] of Reinhart and Rogoff's work [gtod]. Conclusions from the original analysis were adopted as a high profile economic policy driver, raising concerns about the potential impact of analysis errors.

The spectra of approaches to reproducible research are quite broad. In one of the most comprehensive examples, [Brown2012] the authors provide a companion website to their paper where they released their analysis source code, latex paper source, their data, and a turn-key virtual machine-based workflow that allows anyone to regenerate the bulk of the analysis used for the research. In many contexts, each of these steps alone poses a significant challenge. Beyond source code sharing there are several software development environments that support presentation of a computational narrative via a notebook concept. These include IPython [ipython] Notebook, Sage [sage], Matlab, Maple, and Wolfram Mathematica.

Data sharing is also a key component. Systems like the Earth Systems Grid [bernholdt2005earth] have been very successful sharing data, but also require teams to support this sharing. Of course, high performance computing creates additional challenges for data sharing, since the data sets are considerably bigger. (The ESG system faces many of these challenges as a provider of HPC data sets.)

There are many rich visualization frameworks that provide constructs and interface concepts understood by users. For this work, we decided to extend VisIt [HPV_VisIt], in no small part because of its support of Python in its parallelized server [vscipy2012]. Other examples of such frameworks are ParaView [HPV_PV], FieldView [FieldView], and EnSight [EnSight]. From the perspective of a flexible infrastructure for creating custom analyses out of existing primitives, the most comparable work is that of IPython [ipython] and VisTrails [silva2007prov]. Our work is unique in that we have melded a rich visualization framework with a flexible infrastructure for developing new analyses, creating an environment that offers extensibility, usability, and long-term reproducibility.

\section{System}

VisIt is a richly-featured, massively-parallel data analysis and visualization application which runs on hardware ranging from modest desktop systems to large distributed memory compute clusters. VisIt is composed of several cooperating components, each with their own functions within the system. The main component is a central viewer which displays results and acts as a state manager coordinating the different components. Plotted results are generated by a compute server component that reads files, executes data flow networks, and sends results back to the viewer. There are also different clients, including a graphical user interface, Python language interface, and Java language interface. The Python and Java language interfaces allow for complex analysis programs to be built on top of VisIt's infrastructure.

We extended VisIt's existing ability to support multiple simultaneous clients by adding support for Web-based clients, which typically connect on demand. The viewer is able to listen for inbound socket connections from Web clients and establish communication with them using technologies such as WebSockets. We created new proxy classes in various languages such as JavaScript to expose functions that enable a client to control VisIt. These proxy classes enable the creation of lightweight, custom Web applications that dynamically connect to existing VisIt viewer sessions forming the core of the infrastructure needed for collaborative visualization across a range of devices. For example, these enhancements enable VisIt clients running on smart phones and tablet computers to be connected simultaneously to VisIt services running on a shared server.

\section{JSON API}

VisIt normally uses a binary protocol to communicate among components. We enhanced VisIt to also support communication using JavaScript Object Notation (JSON), which allows objects to be represented in an easy to use ASCII form. JSON is widely supported in browsers and Python, eliminating the need for custom client code to transmit and decode VisIt's binary protocol. Using JSON as the mechanism for exchanging objects between VisIt and Web clients enables other novel capabilities. For instance, since JSON objects also communicate the names of fields in addition the field values, we can traverse the JSON objects to automatically create input property panels or provide automatically generated classes.

\section{Scripting API}

[vscipy2012] introduced VisIt's Python Filter Runtime, which embeds a Python interpreter into each MPI Task of VisIt's compute engine. This functionality allows users to write Python scripts that access low-level mesh data structures within VisIt's distributedmemory parallel pipelines. The initial Python Filter Runtime exposed two of VisIt's building blocks to Python programmers:

1) Python Expressions, filters which calculate derived quantities on an existing mesh. 
2) Python Queries, filters which summarize data from an existing mesh.

Building on this infrastructure we extended the use of the Python Filter Runtime into the context of VisIt's Operators, which are filters that implement general data transformations.

This functionality is implemented in a new Scripting Operator and is supported by a Python-based Scripting API. The API allows users to easily compose several Python and R data analysis scripts into a sub-pipeline within VisIt. The goal of this new API is to provide users familiar with Python and R an easier path to embed their analysis within VisIt. To achieve this goal, the Scripting API attempts to shield the user from VisIt's internal filter and contract abstractions and places a focus on writing streamlined analysis routines. This is in contrast to VisIt's Python Expressions and Queries, which require users to understand these abstractions to write filters using Python.

Scripting sub-pipelines are coordinated using a Python dataflow network module. Our Scripting infrastructure leverages the dataflow network's filter graph abstraction to insert additional filters which handle data transformations between VisIt's internal VTK based data model the data structures used in scripts. Python user scripts can process both Python wrapped VTK datasets and field values as numpy arrays. The module uses Rpy 2 to execute scripts written in R. In this context numpy arrays are the primary data structure interface between Python and $\mathrm{R}$ scripts. The module also uses a topological sort to ensure proper script execution precedence and provides reference counting and storage of intermediate results. This ensures that user scripts are executed efficiently.

To support distributed-memory parallel algorithms, both Python and R scripts have access to a MPI context. In Python scripts MPI calls are supported via mpi4py [mpi4py]. In R scripts MPI is supported via pbdMPI [pbdMPI].

We also provide a set of filters that encapsulate common data access patterns for ensemble and time series analysis. These filters are invoked using three categories of script calls: template functions, helper functions, and visit functions:

1) Template functions: for_each_location - at each location call a user defined kernel (written in R or Python) with the data value and a neighborhood around the data point. After kernel execution, the resulting values are returned back to calling script.

2) Helper functions: visit_write - write dataset to a file using a supported format such as NETCDF or visit_get_mesh_info, then return details about the underlying mesh dataset.

3) Visit functions: VisIt operators and utility functions can be registered with the scripting system. Therefore, within the Python or R environment, users can exercise any registered VisIt function and have it return results. For example, the PeaksOverThreshold Operator in VisIt can register a signature with the Script operator and then a user can call this functionality within their script.

\section{Reproducibility}

Each of the clients connected to the VisIt viewer can send commands and state intended to drive the VisIt session. These multiple input streams are consolidated into a single input stream in the viewer that lets the different clients perform actions. As actions are performed, they can cause changes in state that need to be sent
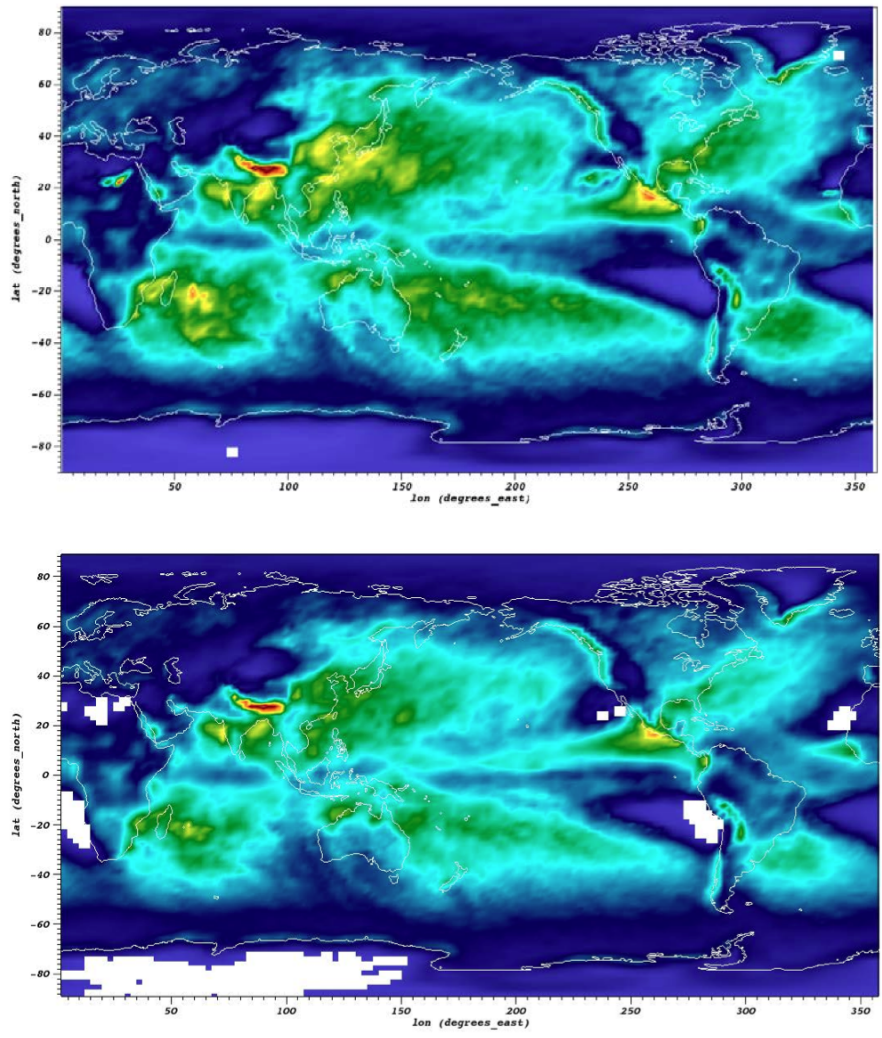

Fig. 1: Extreme precipitation analysis done on an ensemble of two CAM5.1 control runs over 1959-2007 using Generalized Extreme Value Analysis (Top), and Peaks-over-Threshold (Bottom)

back to clients. When new state is sent back to the various clients, they are free to respond as required, depending on their function. For example, when the GUI receives new state, it updates the controls in its windows to reflect the new state from the viewer. When the Python interface receives new state, it transforms the state back into the requisite Python commands needed to cause the state change and logs the commands to a log file that can be replayed later. This same infrastructure is used to record actions taken by the GUI into corresponding Python code that can reproduce the same GUI actions. We have extended VisIt's Python recording mechanism so it annotates the generated Python code with the identity of the user who caused the command to be generated. This increases the available visualization provenance information while still producing a log file that can be replayed to restore the state of the system in a future VisIt session. VisIt's existing Python interface can be used to replay the generated script. We have also extended VisIt's Python interface with a new Writescript () function that can write Python code to reproduce the exact state of the visualization system. This produces Python code that is much more concise, requiring far fewer visualization operations to be performed to restore VisIt's state. We envision being able to build on this capability to automatically produce streamlined domainspecific applications that can set up their plots based on the output from the WriteScript() function.

\section{Evaluation}

The collaboration we have had with climate scientists has proven to be a rich test-bed for the exploration of this workflow. The collaboration began with the integration of VisIt and R to do parallel statistical analysis on very large climate data sets using large 
HPC resources. The climate scientists were interesting in using a statistical technique called extreme value analysis [coles-2001] to understand rare temperatue and precipitation patterns and events in global simulations at very fine temporal resolutions. Initially, several different extreme value analysis algorithms were implemented and incorporated into VisIt as built-in operations. As we worked with the climate scientists, and statisticians, it became clear that a more flexible framework where arbitrary analyses could be easily scripted and experimented with would prove valuable. It would also make it easier for scientists to collaborate, verify various techniques, and make reproducibility much easier.

Figure 1 shows early results using this new framework on estimated annual return values that would occur once every 20 years on average, using Generalized Extreme Value, and Peaks-overThreshold, respectively. The analyses were done on an ensemble of two CAM5.1 control runs over the period of 1959-2007 of daily precipitation.

These analyses required a kernel to be executed at each spatial location using precipitation values over all of the time steps. This was supported using the API call ForEachLocation(user-kernel). The VisIt infrastructure parallelizes the computation required to read in all of the time steps, and aggregates all the time values for each location. The user supplied kernel is then executed using the vector of time-values as input. Another API call is made to write the analysis results out in the desired format, in this case, NETCDF. For both the examples shown in Figure 1, the same API call was made with different user-defined kernels.

Using this capability has several advantages. First, it makes it much easier for domain scientists to experiment with different analysis techniques. Large, parallel visualization frameworks are complex, large pieces of source code, and domain scientists will rarely have the experience to make changes to perform the analysis. This framework allows the scientists to focus on the environment they are most familiar with, analysis kernels written in $\mathrm{R}$ or Python, and leave the details of efficient parallel processing of large scientific data to the visualization framework developers. And second, it makes comparison and reproducibility much easier since the required elements are just the $\mathrm{R}$ or Python kernel code written by the domain scientists. The results can be shared and verified independent of VisIt by execution of the kernel in either Python or R environments on the same, or additional data.

\section{Conclusions and Future Work}

Reproducibility is an important element of the scientific method, since it enables the confirmation of experimental trials that confirm or deny a hypothesis, and visualization is a common mechanism for evaluating experiments. Hence, it is important that visualizations be carried out in a reproducible manner. With this work, we demonstrated that it is possible to extend a richly featured visualization framework with flexible analysis routines in a way that supports reproducibility, and we also demonstrated how capable such a system can be. Further, we considered the problem of collaborative analysis, which is increasingly needed as scientific teams are more and more often made up of large teams. Python was a key element to our success. Since many packages already have Python interfaces, it expedited incorporation of packages like $\mathrm{R}$, and provided a familiar setting for users wanting to develop new interfaces. In total, we believe this work was impactful, since it extends the capabilities of many user groups and does it in a reproducible way. Finally, there are many future directions for this effort, including improved support for plotting and data retrieval (i.e., file readers), language support beyond Python, and tighter integration with the overall VisIt system.

\section{REFERENCES}

[silva2007prov]

Silva, Claudio $\mathrm{T}$ and Freire, Juliana and Callahan, Steven P. Provenance for visualizations: Reproducibility and beyond, Computing in Science \& Engineering 82-89, 2007, IEEE.

[vscipy2012]

Harrison, Cyrus and Krishnan, Hari. Python's Role in VisIt, Proceedings of the eleventh annual Scientific Computing with Python Conference (SciPy 2012).

[gtod] Reinhart, Carmen M. and Rogoff, Kenneth S. Growth in a Time of Debt, American Economic Review, 573-78, September, 2010

[ipython] Perez, Fernando and Granger, Brian E., IPython: a System for Interactive Scientific Computing, Comput. Sci. Eng., 21-29 May, 2007.

[sage] W.A. Stein and others, Sage Mathematics Software, http://sagemath.org

[repo_research_intro] Fomel, S. and Claerbout, J.F. Guest Editors' Introduction: Reproducible Research, Computing in Science Engineering 2009, pages 5-7.

[herndon_debt] Herndon, Thomas and Ash, Michael and Pollin, Robert Does High Public Debt Consistently Stifle Economic Growth? A Critique of Reinhart and Rogoff, April, 2013

[Brown2012] Brown, C Titus and Howe, Adina and Zhang, Qingpeng and Pyrkosz, Alexis B and Brom, Timothy $\mathrm{H}$ A Reference-Free Algorithm for Computational Normalization of Shotgun Sequencing Data, 2012, http: //arxiv.org/abs/1203.4802

[web_repro] Pieter Van Gorp and Steffen Mazanek. SHARE: a web portal for creating and sharing executable research papers, Proceedings of the International Conference on Computational Science, ICCS 2011 589-597, 2011

[res_cloud] Van Gorp, Pieter and Grefen, Paul Supporting the internet-based evaluation of research software with cloud infrastructure, Softw. Syst. Model. 11-28, Feb 2012

[HPV_VisIt] Hank Childs, Eric Brugger, Brad Whitlock, Jeremy Meredith, Sean Ahern, David Pugmire, Kathleen Biagas, Mark Miller, Cyrus Harrison, Gunther H. Weber, Hari Krishnan, Thomas Fogal, Allen Sanderson, Christoph Garth, E. Wes Bethel, David Camp, Oliver Rübel, Marc Durant, Jean M. Favre, and Paul Navrátil. VisIt: An End-User Tool For Visualizing and Analyzing Very Large Data, High Performance Visualization-Enabling Extreme-Scale Scientific Insight, 357372 , Oct 2012

[HPV_PV] Utkarsh Ayachit, Berk Geveci, Kenneth Moreland, John Patchett, and Jim Ahrens, The ParaView Visualization Application, High Performance Visualization-Enabling Extreme-Scale Scientific Insight, 383400, Oct 2012

[EnSight] EnSight User Manual, Computational Engineering International, Inc. December, 2009

[FieldView] Steve M. Legensky. Interactive investigation of fluid mechanics data sets, VIS '90: Proceedings of the $1 \mathrm{st}$ conference on Visualization ' 90 435-439, San Francisco, California, IEEE Computer Society Press

[bernholdt2005earth] Bernholdt, David and Bharathi, Shishir and Brown, David and Chanchio, Kasidit and Chen, Meili and Chervenak, Ann and Cinquini, Luca and Drach, Bob and Foster, Ian and Fox, Peter and others, The earth system grid: Supporting the next generation of climate modeling researc, Proceedings of the IEEE, 485-495, 2005

[pbdMPI] Wei-Chen Chen and George Ostrouchov and Drew Schmidt and Pragneshkumar Patel and Hao Yu, $p b$ dMPI: Programming with Big Data: Interface to MPI, 2012

[mpi4py] Dalcín, Lisandro and Paz, Rodrigo and Storti, Mario and D'Elía, Jorge, MPI for Python: Performance improvements and MPI-2 extensions, J. Parallel Distrib. Comput., May, 2008 
[coles-2001] Stuart Coles, An Introduction to Statistical Modeling of Extreme Values, Springer-Verlag, 2001 